\title{
The Effect of Securing the Mediolateral Stability of the Foot on the Dynamic Balance and Gait of Chronic Stroke Patients Using an Ankle Brace
}

\author{
Daehwan Lee ${ }^{1}$, Jungho Lee ${ }^{2}$ and Younbum Sung ${ }^{3 *}$ \\ ${ }^{1,3}$ Professor, 36616, Department of Physical Therapy, Andong Science College. 189, \\ Seoseon-gil, Seohu-myeon, Andong-si, Gyeongsangbuk-do, Republic of Korea \\ ${ }^{2}$ Professor, 219-705, Department of Physical Therapy, Kyungdong Univ., Bongpo-ri, \\ Toseong-myeon, Goseong-gun, Gangwon-do, Republic of Korea \\ 1dhlee8510@naver.com, ${ }^{2}$ ljhcivapt@naver.com, ${ }^{3 *}$ playeryoon@naver.com
}

\begin{abstract}
Stroke patients have high foot instability and a high risk of falling down in walking and standing positions due to muscle and sensory disorders. There are many patients who complain of inconvenience in their daily lives, but independent walking is very difficult. In this study, the effect of walking and dynamic balance was explored by securing internal and external stability of the foot. A total of 15 patients participated in the experiment, with $10 \mathrm{~m}$ walk tests, timed and go tests, and functional reach tests. The data were statistical comparisons through a paired $t$ test. The walk $10 \mathrm{~m}$ test was not statistically significant, with $19.96 \pm 8.86$ seconds before the device was worn and $19.50 \pm 9.06$ seconds after the device was worn. Time-out and Go tests were statistically significant $24.53 \pm 8.53$ seconds before wearing the device, $20.96 \pm 7.69$ seconds after wearing the device, $23.65 \pm 3.58 \mathrm{~cm}$ before wearing the device, and $27.12 \pm 4.21$ $\mathrm{cm}$ after wearing the device. Securing internal and external stability of the foot can have a positive impact on securing a dynamic balance in which weight movement is performed.
\end{abstract}

Keywords: Foot stability, Dynamic balance, Gait, Foot instruments

\section{Introduction}

A stroke occurs due to ischemic infarction in the brain and cerebral hemorrhage. Stroke patients have difficulty living independently due to severe physical restrictions on movement caused by chronic disabilities in areas such as motor function, cognition, perception, and sensation. Impaired balance strategies and proprioceptive sensations are observed in more than $60 \%$ of stroke patients. Many patients also exhibit decreased or abnormally increased muscle tension, increased postural instability, and decreased movement efficiency. As a result, their static and dynamic balance is compromised, increasing the risk of falls and decreasing their walking speed [1].

Normal and rhythmic walking is achieved through sensory feedback and cooperation of muscles. Stroke patients experience impairments in balance and walking due to muscle issues, such as stiffness or relaxation of the feet. Abnormalities in proprioceptive and tactile senses that receive signals from the ground when standing or walking impede normal walking not only on irregular grounds but also on flat surfaces. Stroke patients are at a higher risk of injuries due

Article history:

Received (January 27, 2021), Review Result (March 2, 2021), Accepted (April 1, 2021) 
to accidents, such as falls, due not only to reduced walking speed but also to reduced accuracy caused by impaired sensory feedback. Sensory feedback transmits exclusive sensory signals to the sensory cortex of the brain and affects even the motor area to regulate the tension of the asymmetrical muscles of the sole, triggering appropriate new movements to improve the motor sense and postural control, thereby helping to maintain balance and stability [2].

Balance is the ability to maintain one's center of gravity within the base of support and is broadly divided into static and dynamic balance. Static balance refers to balance in stationary postures, such as standing and sitting postures, while dynamic balance refers to balance during movement of the body, such as walking, and posture changes. Healthy adults employ several strategies to maintain balance. As an external force that disturbs the balance increases, the hip joint, ankle joint, and one-step strategies are employed - in that order-to maintain balance. As both the ankle joint and the one-step strategy are based on the modification of the ankle joint, it is necessary to secure its stability [3].

Various assistive devices, such as ankle/foot braces, insoles, and wedges, are used to secure the stability of deformed feet of stroke patients [4]. In most cases, stroke patients have cavus feet with lifted heels, which compromise their stability while they are walking. Such feet also receive reduced sensory feedback because their soles do not make sufficient contact with the ground. Given that stroke patients have difficulty in controlling their balance through sensory feedback when walking or standing, assistive devices help their feet firmly touch the ground and secure their ankles' stability, thereby improving their balance [5]. However, the use of assistive devices remains controversial. Some medical experts traditionally believe that these assistive devices interfere with or reduce the work of the muscles, thus preventing or delaying motor recovery. Most experts, however, agree that such devices are beneficial for muscle recovery [6].

Previous studies have reported that foot braces reduce energy consumption during walking. Although the mechanism of this effect is unclear, Simmons et al., [7] reported that foot braces provided a reduction in energy consumption during 10-meter walk, berg balance, and timed up and go tests. However, Lewallen et al. found that assistive devices did not make a significant difference in gait in terms of kinematic morphology. On the other hand, it has been reported that foot braces help improve gait by improving the standing posture and control motion and by properly aligning the ankle of a deformed foot [8]. In particular, it has been reported that securing the ankle's stability during the stance phase can increase walking speed.

Although it appears that the use of assistive devices has both advantages and disadvantages, for patients suffering permanent damage, such as stroke patients, assistive devices are essential for daily life and training. Therefore, in this study, we applied mediolateral splints on the ankles of stroke patients to secure the mediolateral stability of their feet and investigated the effects on their dynamic balance and gait.

\section{Methods}

\subsection{Research subjects}

For this experiment, outpatients of D hospital, D city, Korea, were recruited. The inclusion criteria were (1) foot drop (equinovarus) deformity with stroke onset more than six months previously, (2) lower limbs classified as Brunnstrom's motor recovery stages 3-5, (3) hemiplegic gait without assistive devices, (4) ability to understand and follow verbal instructions, (5) ability to walk at least 10 meters without assistive devices, (6) no orthopedic diseases in the lower limbs, and (7) a Mini-Mental State Examination, Korean version (MMSE- 
K) score of 24 or higher. The exclusion criteria were (1) visual, auditory, or vestibular disorders and (2) walking or balance problems due to other neurological problems unrelated to a stroke.

Based on the inclusion and exclusion criteria, a total of 15 patients, 10 males and 5 females, were recruited. Their mean age was $60.32 \pm 4.72$ years, their mean height was $168.53 \pm 8.65$ $\mathrm{cm}$, and their mean weight was $65.33 \pm 11.23 \mathrm{~kg}$. The average MMSE-K score was $27.23 \pm$ 1.01 points. Cerebral infarction was the stoke type of all patients, and the average time elapsed from the onset of stroke was $13.23 \pm 3.13$ months.

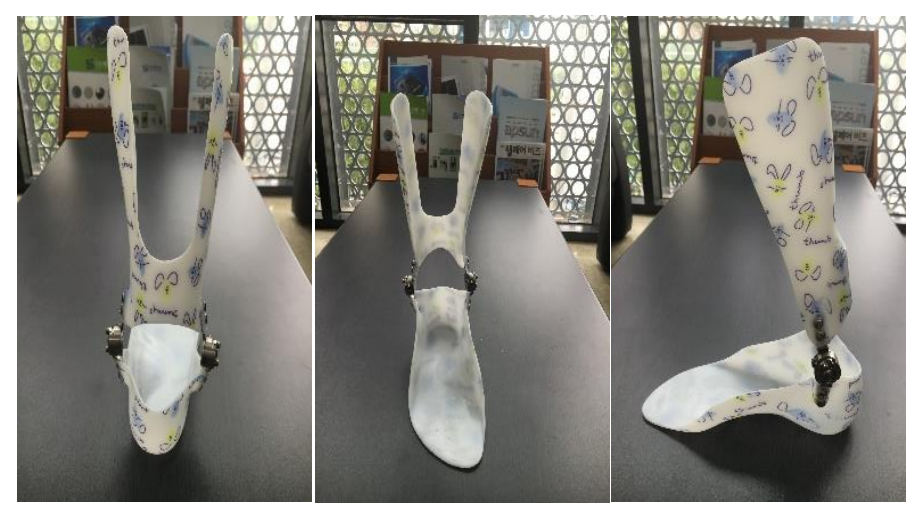

Figure 1. Ankle brace

\subsection{0-meter walk test}

As a tool that can measure the short-distance walking speed and ability of elderly people, this test can also be applied in clinical practice to patients with central nervous system conditions, such as stroke and Parkinson's disease. Each patient was instructed to walk a total of 14 meters without using a cane or walker. To evaluate the gait cycle, a distance of $10 \mathrm{~m}$ was measured, excluding $2 \mathrm{~m}$ at the beginning and $2 \mathrm{~m}$ at the end. To prevent a possible fall, the test was conducted under close supervision by an examiner. Each patient was instructed to perform the test three times, and the average of the three measurements was calculated.

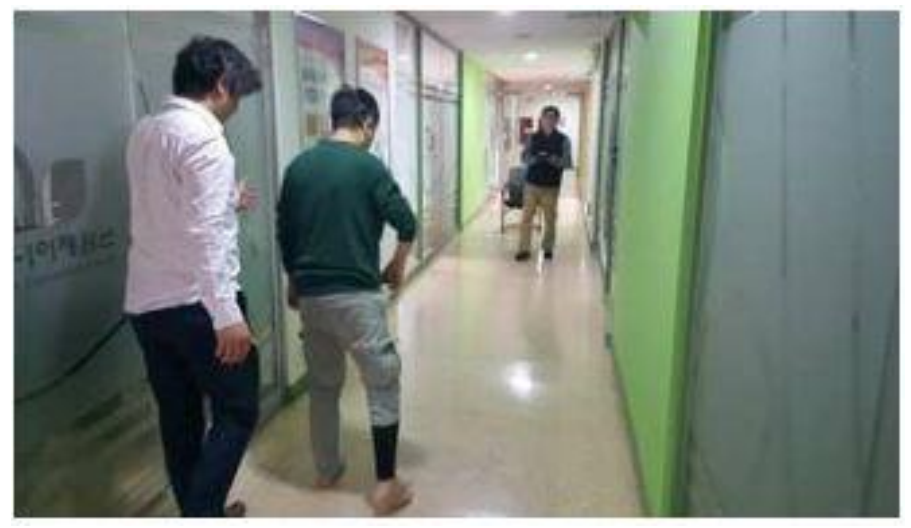

Figure 2. $10 \mathrm{~m}$ walk test

\subsection{Functional reach test}


The functional reach test is an evaluation tool for identifying problems related to balance and estimating the risk of falling in elderly people and has high inter-rater reliability. In this test, each patient stood beside a wall with both feet shoulder-width apart, the 3th fist of the dominant arm lightly clenched, and the shoulder bent 90 degrees, ensuring that the shoulder did not touch the wall. The patient extended the arm to the front as far as possible without moving either foot, and the point where the end of the third metacarpal bone reached was measured. To prevent a possible fall, this test was also performed under close supervision. The test was performed three times, and the average of the measurements was calculated.

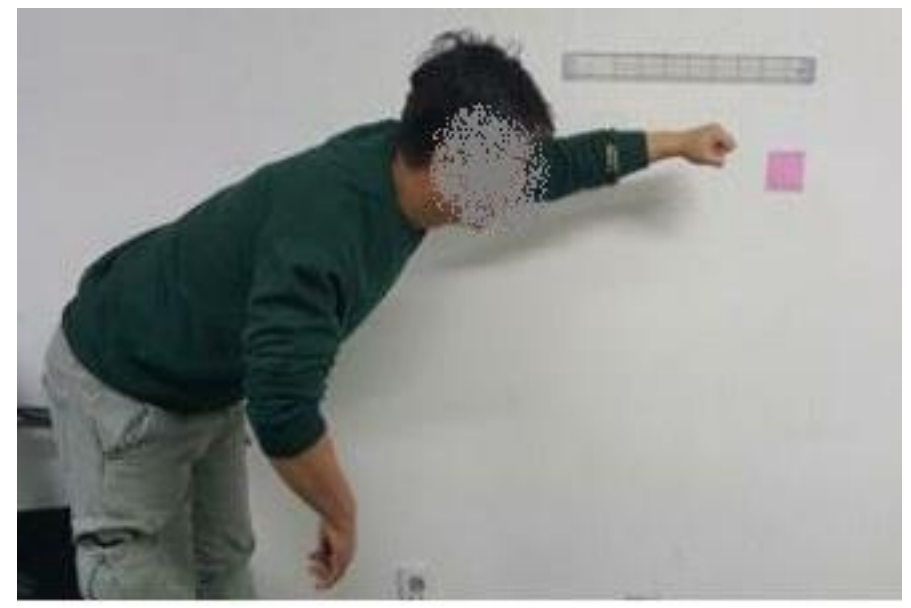

Figure 3. Functional reach test

\subsection{Timed up and go test}

As a tool for evaluating balance ability and the risk of falling, this test can measure both static and dynamic balance. Each patient was seated in a chair and, on the examiner's instructions, walked to a point $3 \mathrm{~m}$ away and back to the chair without using a cane or walker. The time from getting out of the chair to sitting back on it was measured. A healthy adult can complete the test within $10 \mathrm{~s}$. A patient who can complete it within $20 \mathrm{~s}$ belongs to the threshold level of weak elderly people or disabled patients. More than $20 \mathrm{~s}$ indicates that the patient needs help from others while walking, and more than $30 \mathrm{~s}$ indicates that the patient has a very high risk of falling. In this study, each patient performed the test three times, and the average value was used for the analysis. To prevent a possible fall, this test was also performed under close supervision.

\subsection{Data analysis}

Data analysis was performed using the average values of the three measurements for each test. The data were expressed as means \pm standard deviations. A paired t-test was used for comparisons of measurements taken before and while the patients were wearing the ankle brace. The statistical analysis was performed using IBM SPSS Statistics 20.0 for Windows. The swing/stance phase ratio was assessed through descriptive comparisons.

\subsection{Ethical considerations}


The study was conducted in accordance with the ethical standards described in the Declaration of Helsinki. All participants provided written informed consent prior to the study.

For comparative analysis, the patients underwent the 10-meter walk, functional reach, and timed up and go tests before and while wearing ankle braces to secure the mediolateral stability of the foot without controlling or aiding dorsiflexion and plantarflexion.

\section{Results}

\subsection{0-meter walk test}

The mean time to complete the 10-meter walk test was $19.96 \pm 8.86 \mathrm{~s}$ before and $19.50 \pm$ $9.06 \mathrm{~s}$ while wearing the ankle brace. The difference was not statistically significant.

Table $1.10 \mathrm{~m}$ walk test

\begin{tabular}{|c|c|c|c|}
\hline Group & Mean & SD & $p$ \\
\hline Pre & 19.96 & 8.86 & \multirow{2}{*}{0.135} \\
\hline Post & 19.50 & 9.06 & \\
\hline
\end{tabular}

*SD: standard deviation

$* p<0.05 *,<0.01 * *,<0.001 * * *$

*unit: seconds

\subsection{Functional reach test}

The mean reach in the functional reach test was $23.65 \pm 3.58 \mathrm{~cm}$ before and $27.12 \pm 4.21 \mathrm{~cm}$ while wearing the brace. The difference was statistically significant.

Table 2. Functional reach test

\begin{tabular}{|c|c|c|c|}
\hline Group & Mean & SD & $p$ \\
\hline Pre & 236.52 & 35.81 & \multirow{2}{*}{$0.000 * * *$} \\
\hline Post & 271.22 & 42.12 & \\
\hline
\end{tabular}

*SD: standard deviation

$* p<0.05 *,<0.01 * *,<0.001 * * *$

*unit: $m m(10-3 m)$

\subsection{Timed up and go test}

The mean time to complete the timed up and go test was $24.53 \pm 8.53 \mathrm{~s}$ before and $20.96 \pm$ $7.69 \mathrm{~s}$ while wearing the brace. The difference was statistically significant.

Table 3. Timed up and go test

\begin{tabular}{|c|c|c|c|}
\hline Group & Mean & SD & $p$ \\
\hline Pre & 24.53 & 8.53 & \multirow{2}{*}{$0.000^{* * * *}$} \\
\hline Post & 20.96 & 7.69 & \\
\hline
\end{tabular}

*SD: standard deviation

$* p<0.05 *,<0.01 * *,<0.001 * * *$

*unit: seconds 


\section{Discussion}

Stroke patients suffer from weakened muscle strength, cognitive dissonance, and impaired proprioceptive sensation throughout the body, which cause balance, coordination, and functional movement impairments. This study compared the dynamic balance and gait efficiency of stroke patients with foot instability due to weakened muscles and abnormal sensation in their feet before and while wearing an assistive device providing mediolateral stability to their ankles.

Balance is the ability to maintain one's center of gravity within the base of support of the body, that is, the ability to return the center of gravity to the base support of the body without falling, even if the body is moved outside of the base support by an external force or stimulation. This balance is achieved through the coordination and feedback of senses and muscles, which are mostly performed in the subcortical region [9]. With such functions impaired, stroke patients are less capable of controlling their bodies due to abnormal body alignment and muscle tension and slow responses to external stimuli or forces [10].

Foot stability is the first that needs to be established when standing or walking. In the stance phase, if stability is not secured in the process of weight bearing at the time when the hill touches the ground followed by weight being carried over to the next leg, the patient is likely to stagger and, in severe cases, fall [11]. This anxiety is a major aspect of patients' discomfort. In this study, to secure foot stability, the mediolateral stability of the ankle was secured. This resulted in significant differences before and while wearing an ankle brace in the functional reach and timed up and go tests but not in the 10-meter walk test, in which only a small difference was observed. This is because only the mediolateral stability of the foot was ensured, whereas a device that assists muscles necessary for walking was not used. However, securing ankle stability appeared to result in improved performance when the patients stood up and sat down and when they moved the center of gravity outside of the base support. Although there was no statistically significant difference in the 10-meter walk test, this study's findings can still be considered significant, as the walking time was shortened and other gait and balance tests showed significant changes. Taken together, these findings suggest that securing stroke patients' ankle stability with an assistive device while they are walking is important. Unlike our findings, Shin (2017) reported a significant difference in the 10-meter walk test with a Klenzak ankle brace. The Klenzak brace seems to increase flexibility during walking through the auxiliary function of dorsiflexion that it provides. However, it is contraindicated for stroke patients with spastic paralysis.

The $10 \mathrm{~m}$ walk test seems to have a separate problem from securing ankle stability because it is not familiar with the wearing of auxiliary equipment because of its short performance time. When walking, it is possible to increase the performance of walking through the feedback of touch on the soles of the feet as well as visual stimulation, which seems to have been recognized as an obstructive sensation due to the short wearing of assistants.

In this study, descriptive comparisons of gait ratios, step length, and cadence were also performed. Although they hold little statistical significance, it is conceivable that significant results can be obtained with larger sample sizes. Moreover, in this study, only the mediolateral stability of the foot was provided. However, if strength and gait training is combined with using the sensory feedback of the sole during walking through sensory stimulation training of the soles, this could provide a valuable training and daily life support tool for chronic stroke patients. Furthermore, better performance can be achieved if a device capable of providing not only mediolateral stability but also functional support is used in future studies. 
Certain limitations of this study should be noted. Due to the small sample size, the results were greatly influenced by specific. Moreover, variations in the patients' living environments could not be considered because the comparisons were made among outpatients. Future studies that control specific variables could produce more robust results.

\section{Conclusion}

In this study, we investigated the effect of securing the mediolateral stability of the ankle on the performance of balance in patients with stroke. The internal and external stability of the ankle was provided as an instrument, so the coordination of the patient's musculoskeletal system was provided manually by external forces. There were statistically significant differences in functional reaching test and timed up and go test, but no significant values were shown in the $10 \mathrm{~m}$ walking tests. However, we indirectly confirm that securing ankle stability has a positive effect on the performance of static and dynamic balances. Although it is difficult to generalize the findings due to the small sample size and several uncontrolled variables, this study shows that securing the mediolateral stability of the foot can have a positive effect on the dynamic balance of stroke patients. In the future, providing patient ankle stability and training through easy-to-adapted equipment will greatly benefit from improving the performance.

\section{Acknowledgements}

This work was supported by the National Research Foundation of Korea (NRF) grant funded by the Korea government (MSIT) (No- 2020R1I1A1A01071680).

\section{References}

[1] H. Abe, A. Michimata, K. Sugawara, and N. Sugaya, "Improving gait stability in stroke hemiplegic patients with a plastic ankle-foot orthosis,” Tohoku J Exp Med, vol.218, pp.193-199, (2009)

[2] B. J. Darter and J. M. Wiken, "Gait training with virtual reality-based real-time feedback: Improving gait performance following transfemoral amputation,” Physical Therapy, vol.91, no.9, pp.1385-94, (2011)

[3] Y. H. Park, Y. M. Kim, and B. H. Lee, "An ankle proprioceptive control program improves balance, gait ability of chronic stroke patients,” Journal of Physical Therapy Science, vol.25, no.10, pp.1321-1324, (2013)

[4] L. A. B. Ferreira, H. P. Neto, L. A. C. Grecco, T. C. L. Christovão, N. A. Duart, R. D. Lazzari, M. Galli, and C. S. Oliveira, "Effect of ankle-foot orthosis on gait velocity and cadence of stroke patients: A systematic review,” J Phys Ther Sci, vol.25, no.11, pp.1503-1508, (2013)

[5] D. C. Kerrigan, M. E. Karvosky, and P. O. Riley, "Spastic paretic stiff-legged gait: Joint kinetics," American Journal of Physical Medicine and Rehabilitation, vol.80, no.4, pp.244-249, (2001)

[6] F. T. Sarah and M. K. Ruth, "Effects of an ankle-foot orthosis on balance and walking after stroke: A systematic review and pooled meta-analysis," American Congress of Rehabilitation Medicine, vol.94, pp.1377-1385

[7] C. D. Simons, E. H. van Asseldonk, H. van der Kooij, A. Geurts, and J. H. Buurke, "Ankle-foot orthoses in stroke: Effects on functional balance, weight-bearing asymmetry and the contribution of each lower limb to balance control," Clin Biomech (Bristol, Avon), vol.24, pp.769-775, (2009)

[8] J. Lewallen, J. Miedaner, S. Amyx, and J. Sherman, "Effect of three styles of custom ankle foot orthoses on the gait of stroke patients while walking on level and inclined surfaces," J Prosthet Orthot, vol.22, pp.78-83, (2010)

[9] K. H. Kim and S. H. Jang, "A convergence study on effects of progressive proprioceptive motor program training on proprioception and balance ability in chronic stroke patients," Journal of the Korea Convergence Society, vol.9, no.10, pp.81-91, (2018)

[10] J. H. Carr, R. B. Shepherd, L. Nordholm, and D. Lynne, "Investigation of a new motor assessment scale for stroke patients," Physical Therapy, vol.65, no.2, pp.175-180, (1985) 
The Effect of Securing the Mediolateral Stability of the Foot on the Dynamic Balance and Gait of Chronic Stroke Patients Using an Ankle Brace

[11] H. J. Hillstrom, J. Song, A. P. Kraszewski, J. F. Hafer, R. Mootanah, A. B. Dufour, B. S. Chow, and J. T. Deland, "Foot type biomechanics part 1: Structure and function of the asymptomatic foot," Gait Posture, vol.37, pp.445-451, (2013) 